\title{
Contraintes au développement de clusters : Cas de la «Vallée de l'aluminium » au Saguenay- Lac-Saint- Jean
}

\author{
Thierno Diallo, Ph.D ${ }^{1}$. \\ Département des sciences économiques et administratives, \\ $\mathrm{CRDT}^{2}$ et Laboratoire EMC2 ${ }^{3}$ \\ Université du Québec à Chicoutimi
}

\section{Introduction}

Les grappes industrielles (clusters) sont actuellement l'un des instruments privilégiés de la stratégie canadienne sur l'innovation, du programme ACCORD (Action concertée de coopération régionale et de développement) du Gouvernement du Québec ainsi du plan stratégique d'actions structurantes de plusieurs agences de développement économique régional. Une grappe industrielle se définit comme une concentration géographique de sociétés et d'institutions qui entretiennent des rapports systémiques fondés sur les principes de complémentarité, de similitude et de compétition (Rosenfeld, 1997). C'est donc un outil stratégique qui permet d'agir localement sur des enjeux spécifiques selon une démarche de concertation du milieu, par le développement d'alliances et de réseaux stratégiques.

Contrairement à la vision traditionnelle du développement régional qui repose sur l'idée que le développement économique d'une région provient d'un événement dont l'élément déclencheur est extérieur, cette vision moderne repose sur l'implication des acteurs régionaux et le renouvellement de la notion d'innovation. Cette nouvelle approche considère le développement régional comme un événement endogène plutôt qu'exogène, c'est-à-dire comme une interaction entre les différents acteurs régionaux pour créer un système d'innovation régional dynamique. C'est dans cette optique que les gouvernements, en particulier celui du Québec promeut l'utilisation de grappes industrielles pour le développement économique des régions du Québec.

\section{Québec promeut l'utilisation de grappes industrielles pour le développement économique des régions}

La région du Saguenay-Lac-Saint-Jean (SLSJ) étant reconnue mondialement pour l'importance de sa production d'aluminium primaire, les efforts des gouvernements sont centrés donc autour de la promotion de la grappe industrielle dénommée Vallée de l'aluminium. En effet, dans son budget de mars 2000, le gouvernement du Québec a annoncé un ensemble de mesures incitatives pour le développement des industries de transformation de l'aluminium au SLSJ (prêts sans intérêt, aide financière à la formation de maind'œuvre, crédit d'impôt et congé fiscal). Toutefois, malgré toutes ces mesures d'incitations, la Vallée de l'aluminium n'a pas obtenu les succès escomptés. Jusqu'à présent les résultats obtenus en termes de créations d'entreprises et d'emplois restent très modestes. Pourtant les acteurs régionaux comptent encore beaucoup sur un éventuel 
succès de cette grappe industrielle pour la relance économique régionale. Notre étude identifie quelques contraintes majeures qui se

\section{L'industrie de l'aluminium au SLSJ}

L'industrie de l'aluminium renferme des activités allant de la transformation de l'aluminium primaire (alumineries) à la troisième transformation (produits finis) en passant par la première (produits semi ouvrés) et deuxième transformation (produits intermédiaires). Parmi les acteurs de cette industrie se trouvent également les centres de formation, les centres de recherches sur l'aluminium, les centres de transfert et de liaison technologique et les organismes publics de soutien au développement économique (CLD).

Le SLSJ compte un seul producteur d'aluminium primaire, soit Rio Tinto Alcan, qui emploie plus de 6000 personnes. Rio Tinto Alcan exploite 4 usines pour une capacité globale de production d'aluminium primaire équivalente à plus de $50 \%$ de la capacité de la province du Québec, $45 \%$ de la capacité du Canada, et enfin $5 \%$ de la capacité mondiale. Incontestablement donc Rio Tinto Alcan est très présente et très importante dans la structure économique de la région du SLSJ, en particulier dans l'industrie de l'aluminium. Bref, Rio Tinto Alcan constitue un monopole dans la production d'aluminium primaire au SLSJ. Le terme monopole n'est pas en soi toujours péjoratif puisque la théorie économique approuve l'existence de monopole dans des secteurs à rendement d'échelle croissant. Dès lors, la question qui pourrait se poser est de savoir si la production primaire d'aluminium constitue un secteur à rendement d'échelle croissant? Il est clair que cette production est une activité très intensive en capital. D'abord elle nécessite des investissements importants pour l'implantation de centrales électriques. L'électricité est le nerf de la guerre pour l'industrie de l'aluminium. dressent face à l'expansion de la Vallée de l'aluminium au SLSJ.

Elle intervient à diverses phases de la production et représente environ $35 \%$ des coûts d'opération d'une aluminerie. Ensuite, l'implantation ou la modernisation d'une usine d'électrolyse demande aussi un important volume d'investissements. Il semble donc que ce secteur de production est propice à des rendements d'échelle croissant. Rio Tinto Alcan, dans ses activités de production de l'aluminium primaire, occupe bien sa place et celle-ci devra être confortée dans les années à venir par les deux paliers de gouvernement.

La première transformation de l'aluminium mène à des produits semi-ouvrés qui se retrouvent notamment sous la forme de fils de machine, de tubes de tuyaux, de profilés, de tôles, feuilles et feuillards, de câbles et de pièces moulées. Le plus souvent ce sont les producteurs d'aluminium primaire qui font la première transformation à proximité de leur production primaire. Parfois, cette transformation se fait tout simplement dans l'établissement de la production d'aluminium primaire. Le SLSJ compte très peu d'entreprises dans ce secteur de transformation. Rio Tinto Alcan y possède trois usines de première transformation (barres omnibus, fils machine, tôles) en raison notamment de la production de pièce laminées et tréfilées qui se concentre en périphérie des grandes alumineries. Cela représente près de $42 \%$ de toute la production de semi-ouvrés. Par la nature des produits fabriqués et les technologies à grand volume de production, ce secteur ne nécessite pas une main-d'œuvre nombreuse. Ce qui implique un faible niveau d'emplois dans ce domaine pour la région du SLSJ. Quant aux producteurs de pièces moulées et profilées, ce sont généralement des filiales d'entreprises étrangères et quelques 
PME québécoises qui se retrouvent généralement à proximité des grands marchés, en particulier dans la métropole montréalaise.

\section{La promotion de la grappe industrielle Vallée de l'aluminium devrait normalement permettre l'implantation d'entreprises dans ces secteurs}

Selon Sous-traitance industrielle Québec (STIQ), les secteurs des deuxième et troisième transformations de l'aluminium regroupent plus de 1309 établissements. Les principaux secteurs utilisateurs sont: les produits métalliques (43\%), la machinerie $(21,7 \%)$, le matériel de transport $(10,1 \%)$, le matériel électrique $(5,3 \%)$ et les produits informatiques $(4,0 \%)$. Dans la répartition régionale des établissements manufacturiers utilisateurs d'aluminium en 2002, le SLSJ ne possède que 3,5\% de ces établissements. Comparativement aux régions de Montréal et de la Montérégie qui possèdent respectivement $28,6 \%$ et $20,6 \%$, le SLSJ reste de loin en retard dans le développement de ces secteurs industriels. La promotion de la grappe industrielle Vallée de l'aluminium devrait normalement permettre l'implantation d'entreprises dans ces secteurs grâce aux nombreux programmes d'aide financière (prêts sans intérêt et aides financières à la formation de main-d'œuvre) et avantages fiscaux (crédits d'impôt et congés fiscaux) disponibles aux entreprises qui souhaitent s'installer dans la région. Malheureusement, quelques années après l'initiative de la mise en place de cette grappe industrielle, très peu d'emplois ont été créés dans les secteurs des deuxième et troisième transformations de l'aluminium. Cela, malgré de nombreux acquis en termes de formation et de ressources humaines disponibles et compétentes (UQAC, les Cegeps et les Centres de formation), de centres de recherche (CURAL, CTA, CRDA), de chaires de recherche (CHIP, CISMA, TAMLA), et de centres de transfert et de liaison (CETAL, CHT, CQRDA). Il faut rajouter à toutes ces ressources les organismes publics et parapublics de soutien à l'innovation. Certes, toutes ses ressources sont utiles et participent au dynamisme de la région et sont des atouts indéniables pour un renforcement de sa capacité d'innovation. Cependant, il faut reconnaitre que le décollage de l'industrie de l'aluminium au SLSJ tant attendu tarde à rapidement se concrétiser et rien ne laisse présager d'un meilleur avenir. Dès lors, il est opportun de s'interroger sur les contraintes qui se dressent face au développement de la Vallée de l'aluminium du SLSJ.

\section{Identification des contraintes au développement de la «Vallée de l'aluminium » au SLSJ}

Dans cette section, nous énumérons certaines contraintes ou barrières qui se posent dans l'élaboration de stratégies visant à soutenir les grappes industrielles. Selon plusieurs auteurs ${ }^{4}$, la région du SLSJ demeure encore un simple «embryon de district industriel » malgré d'excellentes conditions d'accueil et de fertilisation. Puisque cette grappe tarde à se

La région du SLSJ demeure encore un simple « embryon de district industriel » mettre en place, nous procédons à un diagnostic de contraintes relatives à son développement.

En premier lieu, le diagnostic qui résulte de l'analyse de l'industrie de l'aluminium au SLSJ à la section précédente laisse ainsi apparaître une grappe industrielle « immature » verticale avec un monopole en amont plutôt que verticale avec un monopsone en aval ou horizontale. En effet, cette grappe industrielle repose sur Rio Tinto Alcan (le monopoleur) 
dans l'offre de la matière première et quelques firmes régionales spécialisées dans des créneaux bien spécifiques (Spectube, Aluco). Une grappe verticale avec un monopsone en aval est une grappe industrielle de type de celle dans le domaine de l'aéronautique à Montréal où Bombardier est le monopsone en aval. Les grappes industrielles horizontales sont du type de la Silicon Valley, où plusieurs entreprises sont dans le même créneau et se font concurrence. Généralement, l'intensité de la concurrence des grappes verticales avec un monopsone en amont est plus faible que celle des grappes verticales avec un monopole en aval ou horizontales. Comme cette intensité de la concurrence est un élément prépondérant dans le gain de compétitivité d'une industrie (Porter, 1990), cela laisse prévoir un degré d'innovation plus faible dans les grappes verticales par rapport aux grappes horizontales. Aussi, la finalité d'un cluster est de mettre en place un système régional d'innovation. Pourtant, le principal moteur de l'innovation est la concurrence. Ce qui fait défaut dans la région su SLSJ, d'où le faible degré d'innovation dans la Vallée de l'aluminium.

\section{La théorie économique évoque certaines difficultés de la mise en place d'une grappe en présence d'une grande multinationale}

En deuxième lieu, nous sommes à même de constater de ce qui précède que la grappe industrielle de l'aluminium au SLSJ repose sur la multinationale Rio Tinto Alcan, qui de plus est verticalement intégrée. Bien que cette entreprise se propose d'aider pour le développement de la grappe industrielle, la théorie économique évoque certaines difficultés de la mise en place d'une grappe en présence d'une grande multinationale. L'essence même d'une grappe industrielle est le partage d'information et la confiance mutuelle entre ses participants. La fluidité de l'information et l'implication des partenaires permet de créer rapidement un système d'innovation. Il est difficile de croire qu'une grande multinationale comme Rio Tinto Alcan qui a des intérêts un peu partout dans le monde puisse partager facilement ses informations. Par ailleurs Rio Tinto Alcan possède des filiales actives dans plusieurs secteurs des deuxième et troisième transformations. Ces secteurs sont ceux des l'emballage pharmaceutique et de soins personnels, de l'emballage alimentaire, de l'automobile, des câbles, des anodes, des produits scientifiques, des composites, des alumines de spécialité et de l'aéronautique. Les présences de Rio Tinto Alcan dans ces secteurs peuvent entrainer des comportements stratégiques compréhensibles dans un monde ou la concurrence devient de plus en plus féroce. Cependant, cela peut nuire à l'intensification des échanges et donc de l'innovation dans la grappe industrielle Vallée de l'aluminium. Dans le cas d'économies faibles, l'association, le réseautage, le transfert de connaissances tacites entre les différents acteurs ainsi que la présence de cercles de travailleurs favorisent l'innovation des entreprises. Plusieurs auteurs pensent que cette politique est plus applicable à de petites firmes spécialisées a cause du niveau de croyance et de coopération requis pour la réussite d'une grappe industrielle. Les grandes compagnies multinationales dominent l'économie actuelle et ces compagnies minent la coopération requise pour être effective.

En troisième lieu des contraintes, notons que les grappes industrielles peuvent s'organiser autour de produits, de procédés, de technologies, de compétences, de ressources humaines, ainsi qu'à partir de nombreuses autres sources suivant les défis, les problématiques et les opportunités régionales. Cependant, la plupart des grappes industrielles qui croissent et qui murissent s'organisent soit autour de produits (monopsone), soit autour de savoir-faire (technologies, compétences, et ressources humaines). Dans le cas de grappes qui s'articulent autour de produits, l'interaction 
des participants de la grappe se fait pour l'offre de services et/ou de produits intermédiaires pour un produit final réalisé par un monopsone local. Dans ce cas, la grappe a beaucoup plus de chance de réussir puisque les acteurs étant conscients de l'interdépendance des activités sont plus à même d'échanger des informations et d'agir pour la réussite de la grappe. Pour illustrer notre affirmation, prenons les exemples de Nokia en Finlande pour les téléphones portables, de Nortel à Ottawa pour les réseaux de télécommunication, d'Airbus à Toulouse pour les avions gros porteurs, et de Bombardier à Montréal pour les avions petits porteurs. Dans le cas de grappes qui s'articulent autour de savoir-faires le partage de l'information entre les acteurs de la grappe est très présent puisque certaines applications sont supportées par d'autres applications et l'interdépendance est très forte. Prenons comme exemples la Silicon Valley en Californie, la photonique à Québec et les jeux vidéos à Montréal.

En quatrième lieu des contraintes se trouve l'éloignement des marchés pour les produits en aluminium finis. La localisation des usines de transformation de l'aluminium diffère selon les différentes catégories de produits et leurs procédés de fabrication. Nous avons déjà noté l'avantage concurrentiel de la région du SLSJ pour la production de pièce laminées puisqu'elle nécessite la présence d'une source d'aluminium liquide. Par contre, pour certains procédés qui nécessitent un approvisionnement en matière premières sous forme d'aluminium solide, cet avantage comparatif disparait. Dans ce dernier cas, il semble être plus avantageux de se trouver tout près des marchés de consommation du produit. C'est le cas notamment des marchés de la construction, de l'emballage, du transport, et de l'aéronautique. De plus, les NTIC remplacent de plus en plus le besoin de proximité spatiale pour les grappes. La position concurrentielle du Québec est soumise à de fortes pressions. D'autres pays offrent aux producteurs des conditions très alléchantes en plus d'une proximité intéressante de gigantesques marchés. C'est le cas notamment de la Chine qui est passée, entre 1997 et 2005, du quatrième au premier rang des grands producteurs mondiaux d'aluminium. En 2005, la capacité de production chinoise a été de 7,5 millions de tonnes, soit 2,7 fois celle du Québec. (Voir annexe 1) (Amérique du Nord $50 \%$ à $22 \%$ de 1973 à 2007)

\section{Remarquons l'absence quasiment de centres de $R \& D$ privés dans la région du SLSJ}

En cinquième lieu des contraintes remarquons l'absence quasiment de centres de $R \& D$ privés dans la région du SLSJ. Il existe qu'un seul centre privé, le CRDA de la compagnie Rio Tinto Alcan. Tous les autres centres sont des centres à caractères publics. Ils sont soit universitaires, soit des centres de liaison et de transfert. Les recherches entreprises dans les centres universitaires sont plutôt à vocation fondamentale. Certes, l'UQAC dispose de chercheurs de hauts niveaux dans ces centres de recherches et ces derniers produisent des travaux de recherche de qualité et reconnus universellement. Néanmoins, il faut reconnaitre que ce n'est pas du ressort de la recherche fondamentale d'innover des produits pour les marchés. Cela est plutôt du ressort de la recherche appliquée entreprise dans les centres privés qui sont orientées pour et vers les marchés pour mettre au point des produits compétitifs.

En sixième lieu des contraintes, mentionnons les «Buy Country Act» qui limitent considérablement les possibilités d'exportation dans les différents pays où les produits en aluminium pourraient être en demande. Par exemple le «Buy America Act» requiert que les produits et services des entreprises publiques américaines proviennent en grande majorité du territoire américain. Ce qui a pour effet d'empêcher les industries dans plusieurs 
marchés de se localiser dans des lieux où la demande est faible. Malgré les ouvertures commerciales dues aux accords de libre échange nord-américain (ALENA) et aux règles de l'OMC, il reste que des barrières à la fois tarifaire et non-tarifaire empêchent l'implantation d'entreprises étrangères au SLSJ pour des exportations aux États-Unis, en Europe et en Asie.

\section{Des barrières à la fois tarifaire et non- tarifaire empêchent l'implantation d'entreprises étrangères au SLSJ pour des exportations aux États-Unis, en Europe et en Asie}

En septième lieu des contraintes, nous avons les facteurs environnementaux qui peuvent devenir très contraignants. Dans un classement récent des industries les plus polluantes en gaz à effet de serre au Québec, celle de l'aluminium primaire se classait première. Dans un monde de plus en plus conscient de son environnement, cette industrie risque de devenir la cible des mouvements environnementaux, et cela au détriment des emplois de qualité qu'elle peut générer. Les avantages environnementaux de l'aluminium se trouvent surtout dans les produits finis qui sont souvent très léger et qui requièrent une faible consommation d'énergie relativement aux autres métaux lourds qui requièrent beaucoup d'énergie. Aussi, la production d'aluminium primaire à partir de produits recyclés prend de plus en plus d'importance. Cette production

\section{Conclusion}

La région du SLSJ compte encore beaucoup sur l'industrie de l'aluminium pour sa croissance économique. Malheureusement, le développement de cette industrie dans toutes ses capacités tarde à se concrétiser. Dans cet article, nous avons répertorié quelques-unes des contraintes majeures qui se dressent face au développement de cette industrie. Pour qui requiert moins de $10 \%$ de l'énergie de la production brute, se retrouve dans la plupart des cas en périphérie des grands centres urbains. Ce qui n'est certainement pas un avantage pour la région du SLSJ. La production mondiale d'aluminium secondaire à partir du recyclage s'est élevée à 7,6 Mt en 2005, soit $20 \%$ de la production totale de ce métal. Dans ce même cadre, nous pouvons mentionner la concurrence de nouveaux métaux qui requiert moins d'énergie pour la production et qui sont plus efficaces en termes de dureté et de légèreté.

En huitième et dernier lieu des quelques contraintes que nous voulons mettre en exergue dans cet article, il y'a la pertinence et l'efficacité des politiques de développement régional. Jusqu'à présent ces politiques d'incitation à la création d'entreprises dans les régions en difficultés économiques n'ont pas donné les résultats escomptés. Récemment, le gouvernement du Québec dans son dernier budget a reconnu et remis en question ces politiques de développement régional en adoptant de nouvelles mesures de soutien à l'activité économique régionale. Il y a aussi la remise en question par certains spécialistes de l'utilisation des grappes industrielles comme instruments de développement économique. En effet, ces politiques créent des phénomènes de surspécialisation, ce qui a pour effet de fragiliser considérablement l'économie en cas de choc puisque ceux-ci sont facilement transmissibles dans ces cas.

surmonter ces contraintes, la compagnie Rio Tinto Alcan, premier employeur de la région, qui offre de très bons emplois et qui est le principal acteur économique de la région, doit davantage participer à la mise en place d'un système régional d'innovation et dépasser les intérêts corporatifs pour permettre un vrai développement de la deuxième et troisième 
transformation de cette industrie dans la région $\mathrm{du}$ SLSJ. Cette implication pourrait certainement profiter autant à la région qu'à la compagnie Rio Tinto Alcan. Les deux paliers de gouvernement doivent aussi continuer à soutenir le développement des compétences dans cette industrie. La région du SLSJ regorge déjà de compétences dans ce domaine qu'il faut maintenir. Il reste à mettre en place des innovations pour le marché. Des mesures de promotion pour faire participer activement l'industrie, pour organiser et fournir les services et pour stimuler l'innovation et l'entreprenariat doivent aussi être mises en place pour le partage de l'information, la coopération en R\&D de nouveaux produits et la reconnaissance de la région du SLSJ comme un lieu incontournable pour la transformation de l'aluminium.

\section{Il reste à mettre en place des innovations pour le marché}

\section{Notes et références}

${ }^{1}$ Je remercie sincèrement Marc-Urbain Proulx et Gilles Bergeron pour leurs précieux commentaires.

${ }^{2}$ Centre de recherche sur le développement territorial. (CRDT)

${ }^{3}$ Laboratoire d'expertise multidisciplinaire en conduite du changement (Laboratoire EMC2)

${ }^{4}$ Moussally, 2003; Hassen, 2005; Proulx, 2006

Ben Hassen, T. (2005), « Où en sommes-nous avec la grappe de l'aluminium ?», Organisations et territoires, vol. 14, no 2, printemps-été, p. 27-32.

Moussally, S. (2003), «La vallée de l'aluminium au Saguenay-Lac-Saint-Jean : les possibilités de sa réalisation et les contraintes à maitriser », Classique UQAC.

Porter, M. (1990), «The Competitive Advantage of Nation », The Free Press, New-York.

Proulx, M.-U. (2006), « Le Saguenay-Lac-Saint-Jean face à son avenir », Presses de l’Université du Québec.

Rosenfeld, S. A. (1997), «Bringing Business Clusters into the Mainstream of Economic Development», European Planning Studies, 5:1, 3-23

Sous-Traitance Industrielle Québec (STIQ). (2005), http://www.stiq.com 


\section{Publicité}

\section{Doctorat en développement régional}

